\title{
Phase Estimation and Phase Ambiguity Resolution by Message Passing
}

\author{
Justin Dauwels ${ }^{1}$, Henk Wymeersch ${ }^{2}$, Hans-Andrea Loeliger ${ }^{1}$ and Marc \\ Moeneclaey $^{2}$ \\ 1 Dept. of Information Technology and Electrical Engineering, ETH, CH-8092 \\ Zürich, Switzerland. \\ \{dauwels, loeliger\}@isi.ee.ethz.ch, \\ 2 DIGCOM Research Group, TELIN Dept., Ghent University, \\ Sint-Pietersnieuwstraat 41, B-9000 Gent, Belgium \\ \{hwymeersch, mm\}@telin.ugent.be
}

\begin{abstract}
Several code-aided algorithms for phase estimation have recently been proposed. While some of them are ad-hoc, others are derived in a systematic way. The latter can be divided into two different classes: phase estimators derived from the expectation-maximization (EM) principle and estimators that are approximations of the sum-product message passing algorithm. In this paper, the main differences and similarities between these two classes of phase estimation algorithms are outlined and their performance and complexity is compared. Furthermore, an alternative criterion for phase ambiguity resolution is presented and compared to an EM based approach proposed earlier.
\end{abstract}

\section{Introduction}

This paper deals with iterative code-aided algorithms for phase estimation and phase ambiguity resolution in a communications receiver. Coded channel input symbols are transmitted in frames of $L$ symbols. We consider a channel model of the form

$$
Y_{k}=X_{k} e^{j \Theta}+N_{k}
$$

where $X_{k}$ is the coded channel input symbol at time $k \in\{1, \ldots, L\}, Y_{k}$ is the corresponding received symbol, $\Theta$ is the unknown (constant) phase, and $N_{k}$ is white complex Gaussian noise with (known) variance $2 \sigma_{N}^{2}$, i.e., $\sigma_{N}^{2}$ per dimension. For the sake of definiteness, we assume that the channel input symbols $X_{k}$ are M-PSK symbols and are protected by a low-density parity check (LDPC) code. It is convenient to break $\Theta$ into two contributions:

$$
\Theta=\Phi+Q \frac{2 \pi}{M}
$$

with $0 \leq \Phi<2 \pi / M$ and $Q \in\{0, \ldots, M-1\}$. Accordingly, the problem of estimating $\Theta$ can be decomposed in two subproblems: the problem of estimating $\Phi$, referred to as "phase estimation", and the problem of determining $Q$, which is called "phase ambiguity resolution". 
Several turbo-synchronization algorithms have recently appeared that deal with constant phase rotations [1]-[5]. In [1], phase estimators are derived from the expectation-maximization (EM) principle. In [2], [3] and [4], phase estimation algorithms are presented that are approximations of the sum-product message passing algorithm applied to factor graphs of the channel model. An EM based algorithm for phase ambiguity resolution is proposed in [5].

In this paper, we compare the sum-product based phase estimators of [3] to the EM based phase estimator [1]. To this end, we formulate also the EM based estimator as a message passing algorithm. We refer to [6] for a classical exposition of the EM algorithm. We then compare the EM based phase estimator to several sum-product based algorithms in terms of performance and complexity. We also propose an alternative criterion for phase ambiguity resolution and compare it to the one proposed by Wymeersch et al. [5].

This paper is structured as follows. In Section 2, we briefly explain the factor graph we use to represent channel (1). In Section 3, we review both types of phase estimators and elaborate on the main differences between them. Section 4 considers the problem of phase ambiguity resolution. In Section 5, we investigate the computational complexity of the various estimation algorithms. In Section 6 , we present simulation results.

\section{Factor Graphs of the channel model}

We use Forney-style factor graphs (FFG), where nodes (boxes) represent factors and edges represent variables. A tutorial introduction to such graphs is given in [7]. The system described in Section 1 is easily translated into the FFG of Fig. 1, which represents the factorization of the joint probability function of all variables. The upper part of the graph is the indicator function of the LDPC code, with parity check nodes in the top row that are "randomly" connected to equality constraint nodes ("bit nodes"). The nodes below the bit nodes represent the deterministic mapping $f:\left(B_{k}^{(1)}, \ldots, B_{k}^{\left(\log _{2} M\right)}\right) \mapsto X_{k}$ of the bits $B_{k}^{(1)}, \ldots, B_{k}^{\left(\log _{2} M\right)}$ to the symbol $X_{k}$. These nodes correspond to the factors

$$
\delta_{f}\left(b_{k}^{(1)}, \ldots, b_{k}^{\left(\log _{2} M\right)}, x_{k}\right) \triangleq\left\{\begin{array}{lc}
1, & \text { if } f\left(b_{k}^{(1)}, \ldots, b_{k}^{\left(\log _{2} M\right)}\right)=x_{k} \\
0, & \text { otherwise. }
\end{array}\right.
$$

In Fig. 1, the variable $S$ is defined as $S \triangleq e^{j \Theta}$. The equality constraint node imposes the constraint $S_{k}=S, \forall k$. Furthermore, $Z_{k}$ is defined as $Z_{k} \triangleq X_{k} S_{k}$. The row of "multiply nodes" represents the factors $\delta\left(z_{k}-x_{k} s_{k}\right)$. The bottom row of the graph represents the factors $p\left(y_{k} \mid z_{k}\right) \triangleq\left(2 \pi \sigma_{N}^{2}\right)^{-1} e^{-\left\|y_{k}-z_{k}\right\|^{2} / 2 \sigma_{N}^{2}}$.

\section{Phase Estimation through Message Passing}

Both in the sum-product based algorithms and in the EM based algorithm (which we will also view as message passing in the factor graph), the messages are 


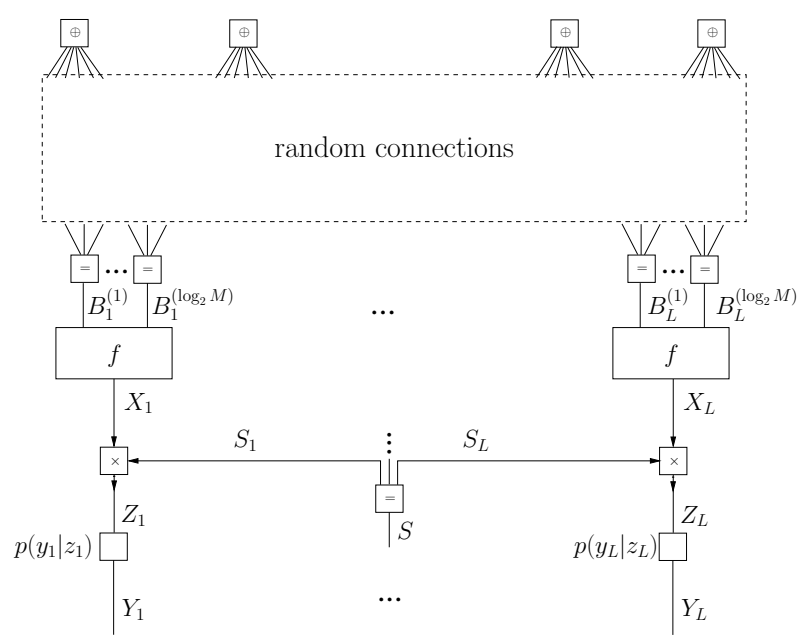

Fig. 1. FFG of LDPC code and the channel model

updated according to the following schedule. First, the bottom row in Fig. 1 is updated, i.e., messages are sent from the received symbols $Y_{k}$ towards the phase model. Then, one alternates between

1. An update of the messages along the $S_{k}$ edges, which can be scheduled as a horizontal (left-to-right) sweep.

2. A subsequent horizontal (left-to-right) sweep for updating both the messages $\mu_{凶 \rightarrow x_{k}}\left(X_{k}\right)$ out of the multiply nodes along the $X_{k}$ edges and the messages $\mu_{f \rightarrow b_{k}}\left(B_{k}\right)$ out of the mapper nodes along the $B_{k}$ edges.

3. Several iterations of the LDPC decoder.

In both algorithms, the messages out of the mapper nodes and inside the graph of the LDPC code are computed according to the standard sum-product rule [7]; we will therefore only consider the computation of the messages related to the phase $\Theta$. First, we briefly elaborate on how the messages are computed according to the sum-product rule, then we consider the EM rule.

Straightforward application of the sum-product rule to compute the messages along the $S_{k}$ edges leads to (intractable) integrals. Several methods to approximate these integral (or, equivalently, to represent the messages along the $S_{k}$ edges) are proposed in [3] and [4]: numerical integration, particle methods, canonical distributions (Fourier, Gaussian and Tikhonov) and gradient methods ("steepest descent"); each of these methods leads to a different phase estimator.

The EM based phase estimator of [1] may be viewed as a message passing algorithm that is similar to the steepest descent sum-product based estimator. In both algorithms, the message $\mu_{S_{k} \rightarrow \bigotimes}\left(s_{k}\right)$ is represented by an estimate $\hat{S}$. In the EM based phase estimator, this estimate is computed according to the 
rule [1]:

$$
\hat{S}=\arg \max _{s} \sum_{i}\left(\sum_{x} \mu_{X_{i} \rightarrow \bigotimes}(x) \mu_{\bigotimes \rightarrow X_{i}}(x) \log \mu_{z_{i} \rightarrow \bigotimes}(x s)\right) .
$$

The rule (4) is similar to the sum-product rule [3]

$$
\hat{S}=\underset{s}{\arg \max } \sum_{i} \log \left(\sum_{x} \mu_{X_{i} \rightarrow \square}(x) \mu_{Z_{i} \rightarrow \bigotimes}(x s)\right) .
$$

Note the difference in the position of the logarithm. In addition, the update rule (4) involves both the incoming messages $\mu_{X_{i} \rightarrow \text { 冈 }}(x)$ and the outgoing messages $\mu_{风 \rightarrow X_{i}}(x)$; in the expression (5), only the incoming messages $\mu_{X_{i} \rightarrow \rrbracket}(x)$ occur. In this particular case, the EM rule can be evaluated analytically [1]. In more complicated problems, this is not the case. One then typically uses steepest descent to compute a hard estimate. In this case, the similarity between EM based and steepest descent sum-product based synchronizers is even stronger.

\section{Phase Ambiguity Resolution}

The algorithms presented in the previous section make estimates of $\Phi$ (see Eq. (2)); they are not able to resolve the phase ambiguity, i.e., to detect $Q$. We determine $Q$ by hypothesis testing. For each possible value of $Q$, we apply the algorithms presented in the previous section. We restrict the domain of the $S_{k}$ messages to the set $\{\exp j \theta: \theta \in[Q 2 \pi / M,(Q+1) 2 \pi / M)\}$. We subsequently select the most likely hypothesis. Wymeersch et al. [5] derived the following EM rule for selecting $Q$ :

$$
\hat{Q}=\underset{q}{\arg \max } \sum_{i}\left(\sum_{x} \mu_{X_{i} \rightarrow \bigotimes}(x) \mu_{\bigotimes \rightarrow X_{i}}(x) \log \mu_{Z_{i} \rightarrow \bigotimes}\left(x \hat{s}_{k}(q)\right)\right),
$$

where $\hat{s}_{k}(q)$ is the estimate of $S_{k}$ under hypothesis $Q$. The rule (6) is an approximation of the MAP detector

$$
\hat{Q}=\underset{q}{\arg \max } \log \sum_{\mathbf{x}} \int_{\phi} p(\mathbf{x}, \mathbf{y}, \phi, q) d \phi .
$$

We propose as alternative approximation

$$
\hat{Q}=\underset{q}{\arg \max } \sum_{i} \log \sum_{x} \mu_{X_{i} \rightarrow \bigotimes}(x) \mu_{\bigotimes \rightarrow X_{i}}(x) .
$$

If the messages $\mu_{S_{k} \rightarrow \bigotimes}\left(s_{k}\right)$ are represented as single values, then the rule (8) reduces to

$$
\hat{Q}=\underset{q}{\arg \max } \sum_{i} \log \sum_{x} \mu_{X_{i} \rightarrow \bigotimes}(x) \mu_{Z_{i} \rightarrow \square}\left(x \hat{s}_{k}(q)\right),
$$


which is very similar to the expression (6). One observes the same differences and similarities between the expressions (6) and (9) as between the expressions (5) and (4). The criterion (8) is more general than (6): the phase messages need not to be represented as single values.

\section{Computational Complexity}

The computational complexity of the phase estimators is directly related to the way the phase messages are represented. Both in the EM algorithm and in the steepest descent sum-product algorithm, the phase messages are single real numbers. As a consequence, the complexity of both algorithms is similar and very low. The complexity of the approach based on numerical integration and particle filtering is much larger: it is proportional to the number of quantization levels and particles respectively, which is typically choosen between 100 and 1000. It is well known that numerical integration becomes infeasable in higher dimensions. Particle filtering on the other hand scales much better with the dimension of the system.

\section{Simulation Results and Discussion}

We performed simulations of the sum-product based as well as the EM based algorithms for joint phase estimation and phase ambiguity resolution. We used a fixed rate $1 / 2$ LDPC code of length 100 that was randomly generated; we did not optimize the code for the channel at hand. The symbol constellation was Gray-encoded 4-PSK. We iterated three times between the LDPC decoder and the phase estimator, each time with hundred iterations inside the LDPC decoder. We did not iterate between the LDPC decoder and the mapper. In the particle filtering (sum-product based) algorithm, the messages were represented as lists of 100 samples; in the numerical integration (sum-product based) algorithm, the phase is uniformly quantized over 100 levels.

Fig. 2 presents the FER (frame error rate) of the presented algorithms for joint phase estimation and ambiguity resolution. We include the FER for perfect synchronization, i.e., for the case in which the phase $\Theta$ is known. Moreover, we show the FER resulting from the M-law phase estimator assuming perfect phase ambiguity resolution. We observe that the M-law estimator gives rise to a degradation of up to $0.5 \mathrm{~dB}$ compared to perfect synchronization. Both the EM estimator as well as the sum-product based estimators are able to reduce most of this degradation. The particle filtering and numerical integration (sum-product based) estimators have slightly lower FER than the EM algorithm and steepest descent sum-product based algorithm, but their complexity is higher.

\section{Acknowledgement}

We heavily used the collection of $\mathrm{C}$ programs for LDPC codes by Radford M. Neal from http://www.cs.toronto.edu/ ${ }^{\sim}$ radford/ldpc.software.html. 




Fig. 2. FER of several phase estimators

This project was in part supported by the Swiss National Science Foundation grant 200021-101955 and the Interuniversity Attraction Pole Program P5/11 Belgian Science Policy.

\section{References}

1. N. Noels et al., "Turbo synchronization : an EM algorithm interpretation", International Conference on Communications 2003, Anchorage, Alaska, May 11-15, 2003, pp. 2933-2937.

2. R. Nuriyev and A. Anastasopoulos, "Analysis of joint iterative decoding and phase estimation for the noncoherent AWGN channel, using density evolution," Proc. 2002 IEEE Information Theory Workshop, Lausanne, Switzerland, June 30 - July 5, 2002, p. 168.

3. J. Dauwels and H. -A. Loeliger, "Phase Estimation by Message Passing," IEEE International Conference on Communications, ICC 2004, Paris, France, June 20-24, 2004, to appear.

4. G. Colavolpe and G. Caire, "Iterative Decoding in the Presence of Strong Phase Noise," IEEE Journal on Selected Areas in Communications, Differential and Noncoherent Wireless Communications, to appear.

5. H. Wymeersch and M. Moeneclaey, "Code-aided phase and timing ambiguity resolution for AWGN channels", The IASTED International Conference, Signal and Image Processing (SIP-03), Honolulu, Hawaii, Aug. 2003.

6. A. P. Dempster, N. M. Laird and D. B. Rubin, "Maximum likelihood from incomplete data via the EM algorithm," Journal of the Royal Statistical Society, 39(1):1-38, 1977, Series B.

7. H. -A. Loeliger, "An introduction to factor graphs," IEEE Signal Processing Magazine, Jan. 2004, pp. 28-41. 